\title{
On the set of hypercyclic vectors for the differentiation operator
}

\author{
Stanislav Shkarin
}

\begin{abstract}
Let $D$ be the differentiation operator $D f=f^{\prime}$ acting on the Fréchet space $\mathcal{H}$ of all entire functions in one variable with the standard (compact-open) topology. It is known since 1950's that the set $H(D)$ of hypercyclic vectors for the operator $D$ is non-empty. We treat two questions raised by Aron, Conejero, Peris and Seoane-Sepúlveda whether the set $H(D)$ contains (up to the zero function) a non-trivial subalgebra of $\mathcal{H}$ or an infinite dimensional closed linear subspace of $\mathcal{H}$. In the present article both questions are answered affirmatively.
\end{abstract}

MSC: $47 \mathrm{~A} 16,37 \mathrm{~A} 25$

Keywords: Hypercyclic operators, Entire functions, Hypercyclic vectors

\section{Introduction}

As usual, $\mathbb{C}$ is the field of complex numbers, $\mathbb{Z}_{+}$is the set of non-negative integers and $\mathbb{N}$ is the set of positive integers. Let $X$ be a topological vector space and $T$ be a continuous linear operator acting on $X$. Recall that $x \in X$ is called a hypercyclic vector for $T$ if the orbit $\left\{T^{n} x: n \in \mathbb{Z}_{+}\right\}$is dense in $X$. By $H(T)$ we denote the set of hypercyclic vectors for $T$. The operator $T$ is called hypercyclic if it has a hypercyclic vector. For more information on hypercyclic operators see the surveys [7, 8 , and references therein. We would just like to mention that the set $H(T)$ for any hypercyclic operator $T$ contains all non-zero vectors from a dense linear subspace of $X$. It follows from the fact due to Bourdon [5] (see also [1]) that if $x \in H(T)$, then $p(T) x \in H(T)$ for any non-zero polynomial $p$. The question whether $H(T)$ for a given operator $T$ must contain all non-zero vectors from a closed infinite dimensional subspace of $X$ was studied by several authors. See [6, 9] for sufficient conditions in terms of the spectrum of $T$ for $H(T)$ to contain all non-zero vectors from an infinite dimensional closed linear linear subspace of $X$ in the case when $X$ is a complex Banach space.

By $\mathcal{H}$ we denote the space of all entire functions $f: \mathbb{C} \rightarrow \mathbb{C}$ with the topology of uniform convergence on compact sets. It is well-known that $\mathcal{H}$ is a Fréchet space. That is, $\mathcal{H}$ is complete metrizable locally convex space, whose topology is defined by the increasing sequence of norms $f \mapsto \max \{|f(z)|:|z| \leqslant n\}$ for $n \in \mathbb{N}$. The differentiation operator

$$
D: \mathcal{H} \rightarrow \mathcal{H}, \quad D f=f^{\prime}
$$

is a continuous linear operator on $\mathcal{H}$. Due to MacLane [10], $D$ is hypercyclic. It is well-known [7] that the set of hypercyclic vectors of any hypercyclic operator on a separable metrizable topological vector space is a dense $G_{\delta}$-set. Hence $H(D)$ is a dense $G_{\delta}$-set in $\mathcal{H}$. We deal with two problems raised by Aron, Conejero, Peris and Seoane-Sepúlveda in [2. It is worth mentioning that $\mathcal{H}$ is an algebra with respect to pointwise multiplication.

Question 1.1. Does $H(D)$ contain all non-zero vectors from a closed infinite dimensional linear subspace of $\mathcal{H}$ ?

Question 1.2. Does $H(D)$ contain all non-constant functions from a non-trivial subalgebra of $\mathcal{H}$ ? In other words, does there exist $f \in \mathcal{H}$ such that $p \circ f \in H(D)$ for any non-constant polynomial $p$ ? 
Note that the analog of the last question for the translation operator $T f(z)=f(z-1)$ on $\mathcal{H}$ has been answered negatively by the same set of authors 3 . Namely, they have shown that for any $f \in \mathcal{H}$ and any $k \geqslant 2, f^{k} \notin H(T)$. In [2] it is also shown that the set $\{f \in \mathcal{H}$ : $f^{n} \in H(D)$ for any $\left.n \in \mathbb{N}\right\}$ is a dense $G_{\delta^{-}}$set in $\mathcal{H}$, thus providing an evidence that the answer to Question 1.2 could be affirmative. In the present paper both above questions are answered affirmatively and constructively. It is worth noting that Question 1.2 was recently independently answered by Bayart and Matheron by means of applying the Baire theorem. Their proof will soon appear in the book [4].

Theorem 1.3. There is a closed infinite dimensional subspace $L$ of $\mathcal{H}$ such that $L \backslash\{0\} \subset H(D)$.

Theorem 1.4. There exists $f \in \mathcal{H}$ such that $p \circ f \in H(D)$ for any non-constant polynomial $p$.

\section{Preliminaries}

Before proving Theorems 1.3 and 1.4, we would like to introduce some notation and mention few elementary facts. Throughout the paper $\mathbb{P}$ stands for the space $\mathbb{C}[z]$ of all complex polynomials in one variable. Clearly $\mathbb{P}$ is a dense linear subspace of $\mathcal{H}$. Let

$$
\mathbb{P}_{0}=\{0\} \text { and } \mathbb{P}_{k}=\{p \in \mathbb{P}: \operatorname{deg} p<k\} \text { for } k \in \mathbb{N}
$$

Obviously $\mathbb{P}_{k}$ is a $k$-dimensional linear subspace of $\mathbb{P}$. If $k \in \mathbb{N}$ and $c>0$, we denote

$$
\mathbb{P}_{k, c}=\left\{p(z)=\sum_{j=0}^{k-1} c_{j} z^{j}:\left|c_{j}\right| \leqslant c \text { for } 0 \leqslant j \leqslant k-1\right\} .
$$

Since we are going to deal with the Taylor series expansion of functions $f \in \mathcal{H}$ rather than their values, we consider a sequence of norms defining the topology of $\mathcal{H}$ different from the one mentioned in the introduction. Namely for $a \in \mathbb{N}$ and $f \in \mathcal{H}$, we write

$$
\|f\|_{a}=\sum_{n=0}^{\infty}\left|f_{n}\right| a^{n}, \quad \text { where } f \in \mathcal{H}, \quad f(z)=\sum_{n=0}^{\infty} f_{n} z^{n} .
$$

It is easy to see that the above sequence of norms is increasing and defines the original topology on $\mathcal{H}$. Moreover, each of these norms is submultiplicative:

$$
\|f\|_{a} \leqslant\|f\|_{b} \text { and }\|f g\|_{a} \leqslant\|f\|_{a}\|g\|_{a} \text { whenever } f, g \in \mathcal{H}, a, b \in \mathbb{N}, a \leqslant b .
$$

Observe that $D\left(\mathbb{P}_{k}\right) \subseteq \mathbb{P}_{k-1}$ for any $k \in \mathbb{N}$. In particular, $D^{n}\left(\mathbb{P}_{k}\right)=\{0\}$ if $n \geqslant k$. Moreover, $\|D p\|_{a} \leqslant \frac{k-1}{a}\|p\|_{a}$ for each $k, a \in \mathbb{N}$ and any $p \in \mathbb{P}_{k}$. Iterating this estimate, we obtain

$$
\left\|D^{n} p\right\|_{a} \leqslant \frac{|(k-n) \ldots(k-1)|}{a^{n}}\|p\|_{a} \leqslant(k / a)^{n}\|p\|_{a} \text { for any } k, n, a \in \mathbb{N} \text { and } p \in \mathbb{P}_{k} .
$$

We also consider the Volterra operator $V: \mathcal{H} \rightarrow \mathcal{H}, V f(z)=\int_{0}^{z} f(t) d t$. It is easy to see that

$$
V f(z)=\sum_{n=1}^{\infty} \frac{f_{n-1}}{n} z^{n}, \text { where } f \in \mathcal{H}, \quad f(z)=\sum_{n=0}^{\infty} f_{n} z^{n}
$$

and that $V$ is a right inverse of $D$. In particular,

$$
D^{n} V^{n}=I \text { for any } n \in \mathbb{N} \text {. }
$$


Using (2.4), one can easily verify that

$$
\left\|V^{n} f\right\|_{a} \leqslant \frac{a^{n}}{n !}\|f\|_{a} \text { for any } n, a \in \mathbb{N} \text { and any } f \in \mathcal{H}
$$

For $f \in \mathcal{H}$, the support of $f$ is the set $\left\{n \in \mathbb{Z}_{+}: f^{(n)}(0) \neq 0\right\}$. Obviously $D$ shifts the supports to the left and $V$ shifts them to the right. That is, if $A$ is the support of $f$, then $A+1=\{n+1: n \in A\}$ is the support of $V f$ and $(A-1) \cap \mathbb{Z}_{+}$is the support of $D f$.

In the proof of Theorems 1.3 and 1.4 we use a sequence in $\mathbb{N} \times \mathbb{P}$ with specific properties.

Lemma 2.1. There exists a sequence $\left\{\left(d_{k}, p_{k}\right)\right\}_{k \in \mathbb{N}}$ of elements of $\mathbb{N} \times \mathbb{P}$ such that

(2.1.1) $d_{k} \leqslant k$ and $p_{k} \in \mathbb{P}_{k, k}$ for each $k \in \mathbb{N}$;

(2.1.2) for any $d \in \mathbb{N}$, the set $\left\{p_{k}: d_{k}=d\right\}$ is dense in $\mathcal{H}$.

Proof. Since $\mathbb{P}$ is dense in $\mathcal{H}$ and $\mathbb{P}$ is the union of $\mathbb{P}_{k, k}$ for $k \in \mathbb{N}$, we can pick a sequence $\left\{s_{j}\right\}_{j \in \mathbb{N}}$ in $\mathbb{P}$ such that $\left\{s_{j}: j \in \mathbb{N}\right\}$ is dense in $\mathcal{H}$ and $s_{j} \in \mathbb{P}_{j, j}$ for any $j \in \mathbb{N}$. It is well-known and easy to see that there is a bijection $\varphi: \mathbb{N} \times \mathbb{N} \rightarrow \mathbb{N}$ such that $\max \{m, j\} \leqslant \varphi(m, j)$ for any $m, j \in \mathbb{N}$. We define a sequence $\left\{\left(d_{k}, p_{k}\right)\right\}_{k \in \mathbb{N}}$ of elements of $\mathbb{N} \times \mathbb{P}$ by the formula

$$
\left(d_{k}, p_{k}\right)=\left(m, s_{j}\right) \text { if } \varphi(m, j)=k .
$$

The condition $\max \{m, j\} \leqslant \varphi(m, j)$ and the obvious inclusion $P_{j, j} \subseteq \mathbb{P}_{k, k}$ for $j \leqslant k$ imply that (2.1.1) is satisfied. Next, let $d \in \mathbb{N}$. From the definition of $\left(d_{k}, p_{k}\right)$ and bijectivity of $\varphi$ it follows that $\left\{p_{k}: d_{k}=d\right\}=\left\{s_{j}: j \in \mathbb{N}\right\}$. Hence $(2.1,2)$ is also satisfied.

\section{Proof of Theorem 1.3}

Let $\left\{\left(d_{k}, p_{k}\right)\right\}_{k \in \mathbb{N}}$ be the sequence of elements of $\mathbb{N} \times \mathbb{P}$ provided by Lemma 2.1. For each $d \in \mathbb{N}$ let $B_{d}=\left\{k \in \mathbb{N}: d_{k}=d\right\}$. By Lemma 2.1, $B_{d}$ are infinite disjoint subsets of $\mathbb{N}$, whose union is $\mathbb{N}$. Let $m_{d}=\min B_{d}$ and $B_{d}^{\prime}=B_{d} \backslash\left\{m_{d}\right\}$. By (2.1.1), $m_{d} \geqslant d$. We also need a sequence increasing fast enough. Namely, pick $\beta: \mathbb{N} \rightarrow \mathbb{N}$ such that

$$
\beta(k+1)>\beta(k)+k \text { and } \beta(k+1)^{\beta(k)} \leqslant 2^{\beta(k+1)} \text { for any } k \in \mathbb{N} .
$$

For each $d \in \mathbb{N}$, we consider the series

$$
f_{d}=g_{d}+\sum_{k \in B_{d}^{\prime}} V^{\beta(k)} p_{k}, \text { where } g_{d}(z)=z^{\beta\left(m_{d}\right)}
$$

Let $a \in \mathbb{N}$. Since $p_{k} \in \mathbb{P}_{k, k}$, we have $\left\|p_{k}\right\|_{a} \leqslant k^{2} a^{k}$. Thus using (2.6), we obtain

$$
\sum_{k \in B_{d}^{\prime}}\left\|V^{\beta(k)} p_{k}\right\|_{a} \leqslant \sum_{k=1}^{\infty}\left\|V^{\beta(k)} p_{k}\right\|_{a} \leqslant \sum_{k=1}^{\infty} \frac{k^{2} a^{k} a^{\beta(k)}}{\beta(k) !}<\infty .
$$

Hence the series defining $f_{d}$ converges absolutely and therefore $f_{d} \in \mathcal{H}$ for $d \in \mathbb{N}$. The inclusions $p_{k} \in \mathbb{P}_{k}$ and the inequality $\beta(k+1)>\beta(k)+k$ imply that the supports of $g_{d}$ and $V^{\beta(k)} p_{k}$ are pairwise disjoint. Hence the supports of $f_{d}$ are pairwise disjoint. It is easy to verify that each sequence of non-zero functions in $\mathcal{H}$ with pairwise disjoint supports is a Schauder basic sequence. Hence $\left\{f_{d}\right\}_{d \in \mathbb{N}}$ is a Schauder basic sequence in $\mathcal{H}$ and the closed linear span $L$ of $\left\{f_{d}: d \in \mathbb{N}\right\}$ consists of the sums of convergent series of the shape $\sum_{d=1}^{\infty} c_{d} f_{d}$ with $c_{d} \in \mathbb{C}$. In order to prove Theorem 1.3, it is enough to demonstrate that $L \backslash\{0\} \subseteq H(D)$. 
Let $f \in L \backslash\{0\}$. Then $f$ is the sum of a convergent series $\sum_{d=1}^{\infty} c_{d} f_{d}$ with $c_{d} \in \mathbb{C}$ being not all zero. Since a non-zero scalar multiple of a hypercyclic vector is hypercyclic, we, multiplying $f$ by a non-zero constant, can assume that there is $b \in \mathbb{N}$ such that $c_{b}=1$. Considering the natural projection onto the subspace of $\mathcal{H}$ of functions whose support is contained in $\left\{\beta\left(m_{d}\right): d \in \mathbb{N}\right\}$, we see that the series $\sum_{d=1}^{\infty} c_{d} g_{d}$ converges in $\mathcal{H}$. Since $g_{d}(z)=z^{\beta\left(m_{d}\right)}$, it follows that $\left|c_{d}\right|^{1 / \beta\left(m_{d}\right)} \rightarrow 0$. In order to verify that $f \in H(D)$ it suffices to demonstrate that

$$
D^{\beta(k)} f-p_{k} \rightarrow 0 \text { in } \mathcal{H} \text { as } k \rightarrow \infty, k \in B_{b} .
$$

Indeed, by Lemma 2.1, $\left\{p_{k}: k \in B_{b}\right\}$ is dense in $\mathcal{H}$. Then (3.2) implies that $\left\{D^{\beta(k)} f: k \in B_{b}\right\}$ is dense in $\mathcal{H}$ and therefore $f \in H(D)$.

It remains to prove (3.2). Let $C=\left\{m_{d}: d \in \mathbb{N}\right\}$. Using definitions of $f$ and $f_{d}$ and the condition $\left|c_{d}\right|^{1 / \beta\left(m_{d}\right)} \rightarrow 0$ it is easy to see that

$$
f=\sum_{d \in \mathbb{N}} c_{d} f_{d}=g+h, \text { where } g=\sum_{d \in \mathbb{N}} c_{d} g_{d} \text { and } h=\sum_{k \in \mathbb{N} \backslash C} c_{d_{k}} V^{\beta(k)} p_{k},
$$

where the series defining $h$ and $g$ are absolutely convergent in $\mathcal{H}$. Let $a \in \mathbb{N}$ and $k \in B_{b}^{\prime}$. Since $D^{n}\left(\mathbb{P}_{j}\right)=\{0\}$ for $n \geqslant j$, we, using the above display together with (2.5), obtain

$$
D^{\beta(k)} f=p_{k}+q_{k}+h_{k}, \text { where } q_{k}=\sum_{m_{d}>k} c_{d} D^{\beta(k)} g_{d} \text { and } h_{k}=\sum_{n \notin C, n>k} c_{d_{n}} V^{\beta(n)-\beta(k)} p_{n} .
$$

Condition $\left|c_{d}\right|^{1 / \beta\left(m_{d}\right)} \rightarrow 0$ implies that there is $c=c(a)>0$ such that $\left|c_{d}\right| \leqslant c(4 a)^{-\beta\left(m_{d}\right)}$ for any $d \in \mathbb{N}$. As we have already mentioned, $\left\|p_{n}\right\|_{a} \leqslant n^{2} a^{n}$ for any $n \in \mathbb{N}$. By (3.1) $\beta(n)-\beta(k) \geqslant n$ for any $n>k$. Hence, using (2.6) and the inequality $\left|c_{d}\right| \leqslant c$, we have

$$
\left\|h_{k}\right\|_{a} \leqslant \sum_{n=k+1}^{\infty} \frac{c n^{2} a^{\beta(n)-\beta(k)+n}}{(\beta(n)-\beta(k)) !} \leqslant \sum_{n=k+1}^{\infty} \frac{c(\beta(n)-\beta(k))^{2} a^{2(\beta(n)-\beta(k))}}{(\beta(n)-\beta(k)) !} \leqslant \sum_{m=k+1}^{\infty} \frac{c m^{2} a^{2 m}}{m !} \rightarrow 0
$$

as $k \rightarrow \infty$. Next, we estimate $\left\|q_{k}\right\|_{a}$. Using the inequality $\left|c_{d}\right| \leqslant c(4 a)^{-\beta\left(m_{d}\right)}$, we obtain

$$
\left\|q_{k}\right\|_{a} \leqslant \sum_{m_{d}>k} c(4 a)^{-\beta\left(m_{d}\right)}\left\|D^{\beta(k)} g_{d}\right\|_{a} \leqslant c \sum_{m_{d}>k}(4 a)^{-\beta\left(m_{d}\right)} \beta\left(m_{d}\right)^{\beta(k)} a^{\beta\left(m_{d}\right)}=c \sum_{m_{d}>k} 4^{-\beta\left(m_{d}\right)} \beta\left(m_{d}\right)^{\beta(k)},
$$

where the last inequality in the above display follows from the equalities $g_{d}(z)=z^{\beta\left(m_{d}\right)}$ and (2.3) . According to (3.1),$\beta\left(m_{d}\right)^{\beta(k)} \leqslant 2^{\beta\left(m_{d}\right)}$ whenever $m_{d}>k$. Substituting these inequalities into the above display we arrive to

$$
\left\|q_{k}\right\|_{a} \leqslant c \sum_{m_{d}>k} 2^{-\beta\left(m_{d}\right)}
$$

It follows that $\left\|q_{k}\right\|_{a} \rightarrow 0$ as $k \rightarrow \infty$. As we already know, $\left\|h_{k}\right\|_{a} \rightarrow 0$ as $k \rightarrow \infty$. Since $a \in \mathbb{N}$ is arbitrary, $q_{k} \rightarrow 0$ and $h_{k} \rightarrow 0$ in $\mathcal{H}$ as $k \rightarrow \infty$. By (3.3), $D^{\beta(k)} f-p_{k} \rightarrow 0$ in $\mathcal{H}$ as $k \rightarrow \infty, k \in B_{b}$. The proof of (3.2) and of Theorem 1.3 is now complete.

\section{Proof of Theorem 1.4}

The main building blocks of our construction of a generator of an algebra contained in $H(D)$ are polynomials of the shape

$$
r_{\alpha, n}(z)=\frac{z^{n}}{n^{n}}+q_{\alpha, n}(z), \text { where } q_{\alpha, n}(z)=n^{(d-1) n} \frac{V^{n^{2}+(d-1) n} p(z)}{d z^{(d-1) n}}, n>1, \alpha=(d, p) \in \mathbb{N} \times \mathbb{P} .
$$


Direct calculations show that

$$
\text { if } p(z)=\sum_{j=0}^{k-1} c_{j} z^{j}, \text { then } q_{\alpha, n}(z)=\frac{n^{(d-1) n}}{d} \sum_{j=0}^{k-1} \frac{j ! c_{j} z^{j+n^{2}}}{\left(j+(d-1) n+n^{2}\right) !} \text {. }
$$

The next lemma is the reason for the choice of $r_{\alpha, n}$.

Lemma 4.1. Let $\alpha=(d, p) \in \mathbb{N} \times \mathbb{P}$ and $a \in \mathbb{N}$. Then

$$
\begin{aligned}
& \lim _{n \rightarrow \infty}\left\|r_{\alpha, n}\right\|_{a}=0, \\
& \text { for any } \nu, b \in \mathbb{N} \text { and } h \in \mathbb{P}, \quad \lim _{n \rightarrow \infty}\left\|D^{\nu}\left(h r_{\alpha, n}^{b}\right)\right\|_{a}=0, \\
& \text { for any } h \in \mathbb{P} \text { and } b \in \mathbb{N}, 1 \leqslant b<d, \quad \lim _{n \rightarrow \infty}\left\|D^{(d-1) n+n^{2}}\left(h r_{\alpha, n}^{b}\right)\right\|_{a}=0, \\
& \lim _{n \rightarrow \infty}\left\|p-D^{(d-1) n+n^{2}}\left(r_{\alpha, n}^{d}\right)\right\|_{a}=0 .
\end{aligned}
$$

Proof. Pick $k \in \mathbb{N}$ such that $p \in \mathbb{P}_{k, k}$. Then $p(z)=\sum_{j=0}^{k-1} c_{j} z^{j}$ with $\left|c_{j}\right| \leqslant k$ for $0 \leqslant j \leqslant k-1$. According to (4.2) and (2.1),

$$
\left\|q_{\alpha, n}\right\|_{a}=\sum_{j=0}^{k-1} \frac{n^{(d-1) n} j !\left|c_{j}\right| a^{j+n^{2}}}{d\left(j+(d-1) n+n^{2}\right) !} \leqslant \sum_{j=0}^{k-1} \frac{k ! n^{(d-1) n} a^{k+n^{2}}}{\left(j+(d-1) n+n^{2}\right) !} \leqslant \frac{2 k ! n^{(d-1) n} a^{k+n^{2}}}{\left(n^{2}\right) !},
$$

where we have used the inequalities $\left|c_{j}\right| j ! \leqslant k$ ! for $0 \leqslant j \leqslant k-1$. Using the Stirling formula, we easily see that $\left(n^{2}\right) ! \geqslant 2\left(n^{2} / e\right)^{n^{2}}$. From this inequality and the above display we see that $\left\|q_{\alpha, n}\right\|_{a} \leqslant k ! a^{k} n^{(d-1) n}(e a)^{n^{2}} n^{-2 n^{2}}$ for $n>1$. Hence there exists $c=c(a, d, p)>0$ such that

$$
\left\|q_{\alpha, n}\right\|_{a} \leqslant(c n)^{-2 n^{2}} \text { for any } n>1 .
$$

By (4.1), $r_{\alpha, n}(z)=(z / n)^{n}+q_{\alpha, n}(z)$. Hence $\left\|r_{\alpha, n}\right\|_{a} \leqslant(a / n)^{n}+\left\|q_{\alpha, n}(z)\right\|_{a}$ and therefore (4.3) follows from (4.7).

According to (4.7), we can pick $c_{1}=c_{1}(a, p, d)>1$ such that $\left\|q_{\alpha, n}\right\|_{a} \leqslant\left(c_{1}-1\right)(a / n)^{n}$ for any $n>1$. Then $\left\|r_{\alpha, n}\right\|_{a} \leqslant(a / n)^{n}+\left\|q_{\alpha, n}(z)\right\|_{a} \leqslant c_{1}(a / n)^{n}$ for any $n>1$. Let $h \in \mathbb{P}$ and $b, \nu \in \mathbb{N}$. Using submultiplicativity of the norm $\|\cdot\|_{a}$, we obtain

$$
\left\|h r_{\alpha}^{b}\right\|_{a} \leqslant c_{1}^{b}\|h\|_{a}(a / n)^{b n} \text { for any } n>1 .
$$

Next, pick $\mu \in \mathbb{N}$ such that $h \in \mathbb{P}_{\mu}$. By (4.1), $h r_{\alpha}^{b} \in \mathbb{P}_{b n^{2}+b k+\mu}$. Thus, applying the above display and the estimate (2.3) , we have

$$
\left\|D^{\nu}\left(h r_{\alpha}^{b}\right)\right\|_{a} \leqslant c_{1}^{b}\|h\|_{a}\left(b n^{2}+b k+\mu\right)^{\nu}(a / n)^{b n} \text { for any } n>1 .
$$

Equality (4.4) follows immediately from the above estimate.

Finally, assume that $1 \leqslant b \leqslant d$. Since $r_{\alpha, n}(z)=(z / n)^{n}+q_{\alpha, n}(z)$, we have

$$
\begin{aligned}
& \left(h r_{\alpha, n}^{b}\right)(z)=\sum_{j=0}^{b}\left(\begin{array}{l}
b \\
j
\end{array}\right) \frac{z^{n j} q_{\alpha, n}(z)^{b-j} h(z)}{n^{n j}}=f_{n}(z)+g_{n}(z), \text { where } \\
& f_{n}(z)=\frac{z^{b n} h(z)}{n^{b n}}+\frac{b q_{\alpha, n}(z) h(z) z^{(b-1) n}}{n^{(b-1) n}} \text { and } g_{n}(z)=\sum_{0 \leqslant j \leqslant b-2}\left(\begin{array}{l}
b \\
j
\end{array}\right) \frac{z^{n j} h(z) q_{\alpha, n}(z)^{b-j}}{n^{n j}} .
\end{aligned}
$$


First, we shall estimate $\left\|g_{n}\right\|_{a}$. Using (4.7) and submultiplicativity of $\|\cdot\|_{a}$, we get

$$
\left\|g_{n}\right\|_{a} \leqslant\|h\|_{a} \sum_{0 \leqslant j \leqslant b-2}\left(\begin{array}{l}
b \\
j
\end{array}\right) \frac{a^{n j}}{n^{n j}}(c n)^{-2(b-j) n^{2}} \text { for any } n>1 .
$$

For $n \geqslant a$ we have $(a / n)^{n j} \leqslant 1$. Since $b-j$ in the above sum is at least 2 , for $n \geqslant c^{-1}$ we have $(c n)^{-2(b-j) n^{2}} \leqslant(c n)^{-4 n^{2}}$. Hence, we can write

$$
\left\|g_{n}\right\|_{a} \leqslant\|h\|_{a}(c n)^{-4 n^{2}} \sum_{0 \leqslant j \leqslant b-2}\left(\begin{array}{l}
b \\
j
\end{array}\right) \leqslant 2^{b}\|h\|_{a}(c n)^{-4 n^{2}} \text { for } n>\max \left\{a, c^{-1}\right\} .
$$

Recall that $h \in \mathbb{P}_{\mu}$ and $p \in \mathbb{P}_{k}$. Then $g_{n} \in \mathbb{P}_{b n^{2}+b k+\mu}$. According to (2.3), $\left\|D^{n^{2}+(d-1) n} g_{n}\right\|_{a} \leqslant$ $\left(b n^{2}+b k+\mu\right)^{n^{2}+(d-1) n}\left\|g_{n}\right\|_{a}$. By (4.8),

$$
\left\|D^{n^{2}+(d-1) n} g_{n}\right\|_{a} \leqslant 2^{b}\|h\|_{a}\left(b n^{2}+b k+\mu\right)^{n^{2}+(d-1) n}(c n)^{-4 n^{2}} \text { for } n>\max \left\{a, c^{-1}\right\} .
$$

Passing to the limit as $n \rightarrow \infty$ we arrive to

$$
\lim _{n \rightarrow \infty}\left\|D^{n^{2}+(d-1) n} g_{n}\right\|_{a}=0 .
$$

Since $\operatorname{deg} q_{\alpha, n}<n^{2}+k$ and $\operatorname{deg} h<\mu$, we see that $\operatorname{deg} f_{n}<n^{2}+k+\mu+(b-1) n-1$. Hence $\operatorname{deg} f_{n}<n^{2}+(d-1) n+k+\mu-(d-b) n$. If $b<d$ and $n \geqslant k+\mu$, we have $\operatorname{deg} f_{n}<n^{2}+(d-1) n$. Hence $D^{n^{2}+(d-1) n} f_{n}=0$. Since $h r_{\alpha, n}^{b}=f_{n}+g_{n}$, we have $D^{n^{2}+(d-1) n}\left(h r_{\alpha, n}^{b}\right)=D^{n^{2}+(d-1) n} g_{n}$ for $n \geqslant k+\mu$ and (4.5) follows from (4.9).

Now consider the case $b=d$ and $h=1$. In this case

$$
f_{n}(z)=\frac{z^{d n}}{n^{d n}}+\frac{d z^{(d-1) n} q_{\alpha, n}(z)}{n^{(d-1) n}} .
$$

Since $n^{2}>n$, we have $n^{2}+(d-1) n>d n$ and therefore $D^{n^{2}+(d-1) n}$ annihilates the first summand in the above display. Since $q_{\alpha, n}(z)=r_{\alpha, n}(z)-(z / n)^{n}$, from (4.1) it follows that

$$
\frac{d z^{(d-1) n} q_{\alpha, n}(z)}{n^{(d-1) n}}=\left(V^{n^{2}+(d-1) n} p\right)(z) .
$$

According to (2.5), we obtain $D^{n^{2}+(d-1) n} f_{n}=D^{n^{2}+(d-1) n} V^{n^{2}+(d-1) n} p=p$. Since $r_{\alpha, n}^{d}=f_{n}+g_{n}$, $p-D^{n^{2}+(d-1) n}\left(r_{\alpha, n}^{d}\right)=-D^{n^{2}+(d-1) n} g_{n}$ and (4.6) follows from (4.9).

We are ready to prove Theorem 1.4. Let $\left\{\left(d_{k}, p_{k}\right)\right\}_{k \in \mathbb{N}}$ be the sequence in $\mathbb{N} \times \mathbb{P}$ provided by Lemma 2.1. Denote $\alpha_{k}=\left(d_{k}, p_{k}\right)$. We shall construct inductively natural numbers $n_{k} \geqslant 2$ such that for any $k \in \mathbb{N}$,

(a1) $n_{k}>n_{k-1}$ if $k \geqslant 2$;

(a2) $\left\|r_{k}\right\|_{k} \leqslant 2^{-k}$, where $r_{k}=r_{\alpha_{k}, n_{k}}$;

(a3) if $k \geqslant 2$, then $\left\|D^{\nu}\left(f_{k}^{j}-f_{k-1}^{j}\right)\right\|_{k} \leqslant 2^{-k}$ for any $j \leqslant k$ and $\nu \leqslant n_{k-1}^{2}+k n_{k-1}$, where $f_{a}=\sum_{l=1}^{a} r_{l}$;

(a4) $\left\|D^{\nu_{k}}\left(f_{k}^{j}\right)\right\|_{k} \leqslant 2^{-k}$ for $1 \leqslant j<d_{k}$ and $\left\|p_{k}-D^{\nu_{k}}\left(f_{k}^{d_{k}}\right)\right\|_{k} \leqslant 2^{-k}$, where $\nu_{k}=n_{k}^{2}+\left(d_{k}-1\right) n_{k}$.

At step 1 we take $n_{1}=3$. Conditions (a1) and (a3) for $k=1$ are trivially satisfied. According to (2.1.1), $d_{1}=1$ and $p_{1}=c$, where $c \in \mathbb{C}$ is a constant, $|c| \leqslant 1$. By (4.1), $r_{1}(z)=\frac{z^{3}}{27}+\frac{c z^{9}}{9 !}$ and therefore $\left\|r_{1}\right\|_{1} \leqslant \frac{1}{27}+\frac{1}{9 !}<2^{-1}$ and (a2) for $k=1$ is satisfied. Since $\nu_{1}=9, d_{1}=1$ and $D^{9} r_{1}=c=p_{1}$, (a4) for $k=1$ is also satisfied. This provides us with the basis of induction. 
Assume now that $m \geqslant 2$ and $n_{k}$ for $1 \leqslant k \leqslant m-1$ satisfying (a1-a4) are already constructed. We have to construct $n_{m}$ satisfying (a1-a4) for $k=m$. We shall actually show that any sufficiently large $n_{m}$ satisfies (a1-a4). For any $n \in \mathbb{N}, n>1$ consider

$$
\rho_{n}=r_{\alpha_{m}, n}, \varphi_{n}=f_{m-1}+\rho_{n} \text { and } \beta_{n}=n^{2}+\left(d_{m}-1\right) n .
$$

Applying Lemma 4.1, we obtain

$$
\begin{aligned}
& \lim _{n \rightarrow \infty}\left\|\rho_{n}\right\|_{m}=0 . \\
& \lim _{n \rightarrow \infty}\left\|D^{\nu}\left(h \rho_{n}^{j}\right)\right\|_{m}=0 \text { for any } \nu, j \in \mathbb{N} \text { and } h \in \mathbb{P} . \\
& \lim _{n \rightarrow \infty}\left\|p_{m}-D^{\beta_{n}}\left(\rho_{n}^{d_{m}}\right)\right\|_{m}=0 \text { and } \lim _{n \rightarrow \infty}\left\|D^{\beta_{n}}\left(h \rho_{n}^{j}\right)\right\|_{m}=0 \text { for any } h \in \mathbb{P} \text { and } 1 \leqslant j<d_{m} .
\end{aligned}
$$

Using the binomial formula, we write

$$
\varphi_{n}^{j}-f_{m-1}^{j}=\sum_{l=0}^{j-1}\left(\begin{array}{l}
j \\
l
\end{array}\right) f_{m-1}^{l} \rho_{n}^{j-l} .
$$

From (4.13) and (4.11) it follows that $\left\|D^{\nu}\left(\varphi_{n}^{j}-f_{m-1}^{j}\right)\right\|_{m} \rightarrow 0$ as $n \rightarrow \infty$ for any $j, \nu \in \mathbb{N}$. Hence

$$
\lim _{n \rightarrow \infty} \max _{\substack{1 \leqslant j \leqslant m \\ 1 \leqslant \nu \leqslant b}}\left\|D^{\nu}\left(\varphi_{n}^{j}-f_{m-1}^{j}\right)\right\|_{m}=0, \quad \text { where } b=n_{m-1}^{2}+m n_{m-1} .
$$

According to (4.13) with $j \leqslant d_{m}$ and (4.12),

$$
\lim _{n \rightarrow \infty}\left\|p_{m}-D^{\beta_{n}} \varphi_{n}^{d_{m}}\right\|_{m}=0 \text { and } \lim _{n \rightarrow \infty} \max _{1 \leqslant j<d_{m}}\left\|D^{\beta_{n}} \varphi_{n}^{j}\right\|_{m}=0 .
$$

It is easy to see that if we set $n_{m}=n$, then $r_{m}=\rho_{n}, \nu_{m}=\beta_{n}$ and $f_{m}=\varphi_{n}$. Thus formulae (4.10), (4.14) and (4.15) imply that (a1-a4) for $k=m$ are satisfied if we choose $n_{m}=n$ being large enough. This completes the inductive construction of the sequence $\left\{n_{k}\right\}$ satisfying (a1-a4).

Condition (a2) and formula (2.2) imply that $\left\|r_{k}\right\|_{m} \leqslant 2^{-k}$ for any $k \geqslant m$. Hence the series $\sum_{k=1}^{\infty} r_{k}$ converges in $\mathcal{H}$ to some $f \in \mathcal{H}$. Equivalently, $f$ is the limit in $\mathcal{H}$ of the sequence $\left\{f_{k}\right\}_{k \in \mathbb{N}}$. In order to prove Theorem 1.4, it suffices to demonstrate that $p \circ f \in H(D)$ for any non-constant polynomial $p$. Since the set $H(D)$ is closed under multiplication by non-zero scalars, it is enough to show that $p \circ f \in H(D)$ if a polynomial $p$ has shape

$$
p(z)=z^{d}+\sum_{j=0}^{d-1} c_{j} z^{j}, \quad d \in \mathbb{N} .
$$

By Lemma 2.1, the set $B=\left\{k \in \mathbb{N}: d_{k}=d\right\}$ is infinite and the set $\left\{p_{k}: k \in B\right\}$ is dense in $\mathcal{H}$. Thus it is enough to prove that

$$
p_{k}-D^{\nu_{k}}(p \circ f) \rightarrow 0 \text { in } \mathcal{H} \text { as } k \rightarrow \infty, k \in B,
$$

where $\nu_{k}=n_{k}^{2}+\left(d_{k}-1\right) n_{k}$. Indeed, if it is the case, then density of $\left\{p_{k}: k \in B\right\}$ in $\mathcal{H}$ implies density of $\left\{D^{\nu_{k}}(p \circ f): k \in B\right\}$ in $\mathcal{H}$ and therefore hypercyclicity of $p \circ f$ for $D$. It remains to prove (4.16). Let $m \in \mathbb{N}$ and $k \in B$ be such that $k \geqslant m$. Since $d_{k}=d$ and $m \leqslant k$, condition (a4) implies

$$
\left\|p_{k}-D^{\nu_{k}}\left(f_{k}^{d}\right)\right\|_{m} \leqslant 2^{-k} \text { and }\left\|D^{\nu_{k}}\left(f_{k}^{j}\right)\right\|_{m} \leqslant 2^{-k} \text { for } 1 \leqslant j<d .
$$


Let $\nu \in \mathbb{N}, \nu \geqslant k$. Then $d=d_{k} \leqslant k \leqslant \nu$ and according to (a3), we have

$$
\left\|D^{\nu_{k}}\left(f_{\nu+1}^{j}\right)-D^{\nu_{k}}\left(f_{\nu}^{j}\right)\right\|_{m} \leqslant 2^{-\nu-1} \text { for } 1 \leqslant j \leqslant d .
$$

Using the triangle inequality and the above two displays, we get that

$\left\|p_{k}-D^{\nu_{k}}\left(f_{n}^{d}\right)\right\|_{m} \leqslant 2^{-k}+\sum_{\nu=k}^{n} 2^{-\nu-1} \leqslant 2^{1-k}$ and $\left\|D^{\nu_{k}}\left(f_{n}^{j}\right)\right\|_{m} \leqslant 2^{-k}+\sum_{\nu=k}^{n} 2^{-\nu-1} \leqslant 2^{1-k}$ for $1 \leqslant j<d$

for any $n \geqslant k$. Since $f_{n} \rightarrow f$ in $\mathcal{H}$ we have $f_{n}^{j} \rightarrow f^{j}$ in $\mathcal{H}$ as $n \rightarrow \infty$ for any $j \in \mathbb{N}$. Therefore passing to the limit as $n \rightarrow \infty$ in the above display, we obtain

$$
\left\|p_{k}-D^{\nu_{k}}\left(f^{d}\right)\right\|_{m} \leqslant 2^{1-k} \text { and }\left\|D^{\nu_{k}}\left(f^{j}\right)\right\|_{m} \leqslant 2^{1-k} \text { for } 1 \leqslant j<d, k \in B, k \geqslant m .
$$

Hence

$$
\left\|p_{k}-D^{\nu_{k}}(p \circ f)\right\|_{m} \leqslant 2^{1-k}\left(1+\sum_{1 \leqslant j<d}\left|c_{j}\right|\right) \text { for } k \in B, k \geqslant m .
$$

Passing to the limit as $k \rightarrow \infty$, we see that $\left\|p_{k}-D^{\nu_{k}}(p \circ f)\right\|_{m} \rightarrow 0$ as $k \rightarrow \infty, k \in B$. Since $m \in \mathbb{N}$ is arbitrary, (4.16) is satisfied. The proof of Theorem 1.4 is now complete.

\section{Remarks}

The function $f$ constructed in the proof of Theorem 1.4 has moderate growth. Namely, it is easy to see that $|f(z)|=O\left(e^{(1+\varepsilon)|z|}\right)$ as $|z| \rightarrow \infty$ for each $\varepsilon>0$. In particular, $f$ has finite exponential type. It is worth noting that a function from $H(D)$ can not have exponential type $<1$ [10]. It is also easy to verify that there can be no common growth restriction for the functions from the space $L$ from Theorem 1.3. We would like to discuss possible modifications of Questions 1.1 and 1.2. Note that $H(D)$ contains (up to the zero function) no non-trivial ideals in $\mathcal{H}$. Indeed, let $f \in \mathcal{H} \backslash\{0\}$. Then the function $g(z)=\overline{f(\bar{z})}$ also belongs to $\mathcal{H} \backslash\{0\}$ and $(f g)(\mathbb{R}) \subseteq \mathbb{R}$. The latter inclusion implies that $f g \notin H(D)$. Indeed the set of functions real on the real axis is closed and nowhere dense in $\mathcal{H}$ and is preserved by $D$. Thus the ideal generated by $f$ contains the non-zero function $f g$, which is not hypercyclic for $D$. Finally we would like to raise the following question.

Question 5.1. Does $H(D)$ contain all non-constant functions from a non-trivial closed subalgebra of $\mathcal{H}$ ? Equivalently, does there exist $f \in \mathcal{H}$ such that $g \circ f \in H(D)$ for any non-constant $g \in \mathcal{H}$ ?

It seems likely that the answer to the above question is negative. To prove this it would be sufficient for any $f \in \mathcal{H}$ to find a non-constant $g \in \mathcal{H}$ and a bounded sequence $\left\{z_{n}\right\}_{n \in \mathbb{Z}_{+}}$in $\mathbb{C}$ such that the sequence $\left\{(g \circ f)^{(n)}\left(z_{n}\right)\right\}_{n \in \mathbb{Z}_{+}}$is bounded. Indeed, boundedness of the last sequence would prevent $g \circ f$ from being hypercyclic for $D$.

Leon and Montes [9] have shown that if $T$ is a continuous linear operator on a Banach space $X$ and $\sigma_{e}(T)$, being the set of $\lambda \in \mathbb{C}$ such that $T-\lambda I$ is not Fredholm, does not intersect the closed unit ball $\{z \in \mathbb{C}:|z| \leqslant 1\}$, then there is no closed infinite dimensional subspaces $L \subset X$ such that $L \backslash\{0\} \subset H(T)$. It is easy to see that $\sigma_{e}(D)=\varnothing$. Thus the above result does not carry through to operators on Fréchet spaces.

Acknowledgements. The author is grateful to the referee for helpful comments and numerous corrections. 


\section{References}

[1] S. Ansari, Hypercyclic and cyclic vectors, J. Funct.Anal. 128 (1995), 374-383

[2] R. Aron, J. Conejero, A. Peris and J. Seoane-Sepúlveda, Sums and products of bad functions. Function spaces, 47-52, Contemp. Math. 435, Amer. Math. Soc., Providence, RI, 2007

[3] R. Aron, J. Conejero, A. Peris and J. Seoane-Sepúlveda, Powers of hypercyclic functions for some classical hypercyclic operators, Integral Equations Operator Theory 58 (2007), 591-596

[4] F. Bayart and E. Matheron, Dynamics of linear operators, Cambridge University Press, 2009

[5] P. Bourdon, Invariant manifolds of hypercyclic vectors, Proc. Amer. Math. Soc. 118 (1993), 845-847

[6] M. Gonzáles, F. Leon-Saavedra and A. Montes-Rodríguez, Semi-Fredholm theory: hypercyclic and supercyclic subspaces, Proc. London Math. Soc. 81 (2000), 169-189

[7] K. Grosse-Erdmann, Universal families and hypercyclic operators, Bull. Amer. Math. Soc., 36 (1999), 345-381

[8] K. Grosse-Erdmann, Recent developments in hypercyclicity, RACSAM Rev. R. Acad. Cienc. Exactas Fis. Nat. Ser. A Mat., 97 (2003), 273-286

[9] F. Leon-Saavedra and A. Montes-Rodríguez, Spectral theory and hypercyclic subspaces, Trans. Amer. Math. Soc. 353 (1997), 247-267

[10] G. MacLane, Sequences of derivatives and normal families. J. Analyse Math. 2 (1952), 72-87

STANisLAV SHKARIN

QueEns's University Belfast

Department of Pure Mathematics

University ROAD, Belfast, BT7 1NN, UK

E-MAIL ADDRESS: s.shkarin@qub.ac.uk 